Supporting Information

\title{
Preparation of Sustainable Polar Aprotic Solvents from Biomass: One-Pot Two- Step Catalytic Reaction of Cellulose with N,N-Dimethylurea over Ru/C
}

Tayyebeh B. Champa ${ }^{a}$, Shuo Liu ${ }^{b}$, Benjamin L. Wegenhart ${ }^{b}$, Tony Zhang ${ }^{* c 1}$, and Mahdi M. Abu-Omar*a

a Department of Chemistry and Biochemistry, University of California, Santa Barbara, Santa Barbara, CA, 93106, USA

b Department of Chemistry, Purdue University, West Lafayette, IN 47907, USA

' Lilly Research Laboratories, Eli Lilly and Company, Indianapolis, IN 46285, USA

*Corresponding authors, email:

abuomar@chem.ucsb.edu

tony@tyligand.com

Pages 8; Figures 1; Tables 5

\section{Summary of content:}

I. Product characterization for compounds TMI and TMHI ................. S2

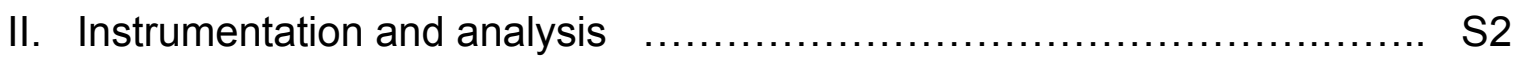

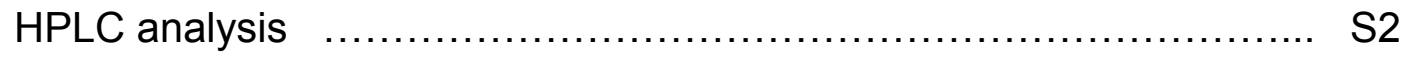

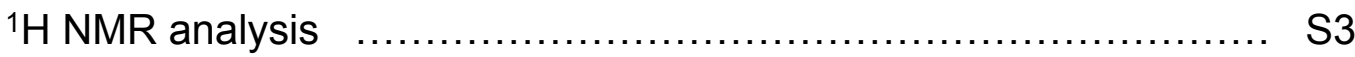

Table S1. The effect of catalyst loading on cellulose conversion and PAS selectivity

Table S2. Mass balance, cellulose and DMU conversion, and PAS product selectivity S5

Table S3. Mass balance, sugar and DMU conversion, and PAS product selectivity S6

Table S4. Results of coupling reactions of 1,2-PG and DMU at various temperature S7

Table S5. Results of in situ formation of homogeneous Ru catalysts with varying phosphine ligands S7

III. Figure S1. ${ }^{1} \mathrm{H}$ NMR of the oxidation of 1,2-propylene glycol S8

\footnotetext{
${ }^{1}$ Current affiliation: Tyligand Bioscience, Shanghai, China.
} 


\section{Product Characterization for Compounds TMI and TMHI}

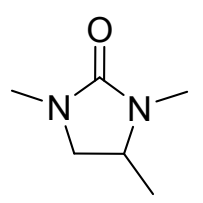

1,3,4-trimethylimidazolidin-2-one (TMI); colorless liquid, ${ }^{1} \mathrm{H}-\mathrm{NMR}\left(400 \mathrm{MHz}, \mathrm{CDCl}_{3}\right)$ $\delta 3.40(\mathrm{~d}, 2 \mathrm{H}, \mathrm{J}=8 \mathrm{~Hz}), 2.76(\mathrm{~m}, 1 \mathrm{H}), 2.74(\mathrm{~s}, 3 \mathrm{H}), 2.71(\mathrm{~s}, 3 \mathrm{H}), 1.20(\mathrm{~d}, 3 \mathrm{H}, \mathrm{J}=8 \mathrm{~Hz})$; ${ }^{13} \mathrm{C}-\mathrm{NMR}(100 \mathrm{MHz}, \mathrm{CDCl} 3) \delta$ 161.3, 52.6, 50.9, 30.9, 28.5, 17.8; MS (El) m/z (rel. intensity): $128\left(\mathrm{M}^{+}, 39\right), 113$ (100), 98 (5), 85 (20), 72 (22), 58 (29), 42 (40)

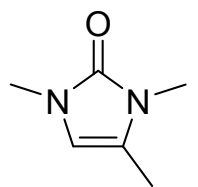

1,3,4-trimethyl-2,3-dihydroimidazol-2-one (TMHI); Pale yellow solid, ${ }^{1} \mathrm{H}-\mathrm{NMR}$ (400 $\left.\mathrm{MHz}, \mathrm{CDCl}_{3}\right) \delta 5.9(\mathrm{q}, 1 \mathrm{H}, \mathrm{J}=1.3 \mathrm{~Hz}), 3.18(\mathrm{~s}, 3 \mathrm{H}), 3.16(\mathrm{~s}, 3 \mathrm{H}), 2.00(\mathrm{~d}, 3 \mathrm{H}, \mathrm{J}=1.3$ $\mathrm{Hz}) ;{ }^{13} \mathrm{C}-\mathrm{NMR}(100 \mathrm{MHz}, \mathrm{CDCl} 3) \delta$ 153.6, 118.4, 106.9, 29.9, 27.1, 10.1; MS (EI) m/z (rel. intensity): $126\left(\mathrm{M}^{+}, 100\right), 125$ (26), 111 (16), 97 (18), 70 (6), 56 (92), 42 (40)

\section{Instrumentation and Analysis}

\section{HPLC analysis}

The selectivity and the yield of the target molecules in DMI, ethanol, Methanol, and water and the remaining amount of DMU were analyzed with Agilent 1260 Infinity Quaternary High-Performance Liquid Chromatography (HPLC) system, using Zorbax Eclipse XDB-C18 Column $(250 \times 74.6 \mathrm{~mm})$ set at $25^{\circ} \mathrm{C}$. The chromatography apparatus is equipped with G1315D Diode Array Detector (DAD). All analysis was done at the wavenumber of $210 \mathrm{~nm}$. A mixture of $\mathrm{H}_{2} \mathrm{O}(\mathrm{A})$ and acetonitrile (B) were used as the mobile phase at a flow rate of $0.5 \mathrm{~mL} / \mathrm{min}$. Nonlinear gradient was used (95\% A and $5 \% \mathrm{~B}$ from beginning to $80 \% \mathrm{~A}$ and $20 \% \mathrm{~B}$ at 30.0 minute and then back to the starting point over 10 minutes). A fixed amount (500 $\mu \mathrm{L})$ of internal standard benzyl alcohol (5.0 mM) was added into each sample for the quantification purposes. Standard curves for all the target products were made by comparison of the products to internal standard. All results were analyzed and quantified according to standard curves. Before analyzing by HPLC, the liquid samples were filtered through a $0.22 \mu \mathrm{m}$ cutoff syringe filter ( $25 \mathrm{~mm}$ diameter).

All sugar and cellulose samples as well as the polyol products from their $\mathrm{C}-\mathrm{C}$ bond cleavage were analyzed with Agilent 1260 Infinity Quaternary High-Performance Liquid Chromatography (HPLC) system, using Aminex HPX-87H column (300 x 7.8 $\mathrm{mm}$ ) set at $70^{\circ} \mathrm{C}$. The chromatography apparatus is equipped with $\mathrm{G} 1362 \mathrm{~A}$ Refractive Index Detector (RID) calibrated with external standards. A $0.005 \mathrm{M}$ sulfuric acid solution was employed as the mobile phase with flow of $0.6 \mathrm{~mL} / \mathrm{min}$. A fixed amount $(500 \mu \mathrm{L})$ of internal standard cis-1,5-cyclooctane diol $(10 \mathrm{mM})$ was added into 
each sample for quantification purposes. Before analyzing by HPLC, the aqueous solutions were filtered through a $0.22 \mu \mathrm{m}$ cutoff syringe filter ( $25 \mathrm{~mm}$ diameter).

Cellulose conversion $=\left(\left(\mathrm{m}_{i}-\mathrm{m}_{d}-\mathrm{m}_{R u / C}\right) / \mathrm{m}_{i}\right)$

$\mathrm{m}_{i}=$ Initial mass of cellulose before the reaction

$\mathrm{m}_{d}=$ mass of dried solid residue (remaining cellulose + catalyst) at $60^{\circ} \mathrm{C}$ overnight after the reaction

$\mathrm{m}_{R u / C}=$ Initial mass of $\mathrm{Ru} / \mathrm{C}$ before the reaction

Polyol selectivity $\%=(\#$ mmoles of carbons in each compound $/ \#$ mmoles of carbons converted in cellulose) * 100

PAS selectivity $\%=(\#$ mmoles of carbons in each compound / \# mmoles of DMU converted) $* 100$

\section{${ }^{1} \mathrm{H}$ NMR Analysis}

${ }^{1} \mathrm{H}$ NMR analysis was performed on either a Varian Mercury-300 instrument equipped with a $5 \mathrm{~mm}$ 4-nucleus/BB probe or a Bruker DRX-500 instrument equipped with a $5 \mathrm{~mm}$ TXI Z-gradient cryoprobe.

${ }^{1} \mathrm{H}$ NMR yields were determined by integration of the produce versus a known quantity of internal standard (naphthalene). The amount of product was calculated by the following equation:

$$
\mathrm{mol}_{\text {prod }}=\mathrm{mol}_{s t d}{ }^{*}\left(\text { Integral }_{\text {prod }} / \text { Integral }\left.\right|_{s t d}\right) *\left(\mathrm{~N}_{s t d} / \mathrm{N}_{\text {prod }}\right)
$$

Where $N$ is the number of nuclei for the corresponding signal. The yield is then determined by the following formula:

$$
\text { Yield }_{\text {prod }} \%=\left(\mathrm{mol}_{\text {prod }} / \mathrm{mol}_{\text {l.r. }}\right) * 100
$$

Where mol $\mathrm{I}_{\text {.r. }}$ is the moles of limiting reactant in the reaction. The selectivity is then defined as:

$$
\text { Selectivity } \%=\left(\operatorname{mol}_{\text {prod }} /\left(\operatorname{mol}_{i(\text { reactant })}-\operatorname{mol}_{f(\text { reactant })}\right)\right) * 100
$$


Table S1. The effect of catalyst loading on cellulose conversion and PAS selectivity. ${ }^{\text {a }}$ $\%$ Cellulose conversion (\% PAS

\begin{tabular}{cccc} 
Catalyst (g) & \multicolumn{3}{c}{ selectivity) } \\
& $\mathrm{H} 2 \mathrm{O}$ & $\mathrm{MeOH}$ & $\mathrm{DMI}$ \\
\hline 0.4 & $46(7)$ & $37(15)$ & $23(35)$ \\
0.2 & $32(5)$ & $21(12)$ & $13(31)$ \\
0.1 & $19(1)$ & $14(5)$ & $8(11)$ \\
\hline
\end{tabular}

aSigmacell cellulose $(2.0 \mathrm{~g}), \mathrm{DMU}(0.2 \mathrm{~g}, 2.23 \mathrm{mmol}), \mathrm{Ru} / \mathrm{C}(0.4,0.2,0.1 \mathrm{~g})$, and solvent (20 $\mathrm{ml}$ ) were heated in a $75 \mathrm{ml}$ stainless steel autoclave at $200^{\circ} \mathrm{C}$ in two steps: first, under $\mathrm{N}_{2}$ inert atmosphere ( $35 \mathrm{bar}$ ) for 6 hours, then $\mathrm{H}_{2}$ (30 bar) for 6 hours. Ru/C has $5 \mathrm{wt} \% \mathrm{Ru}$ content. 
Table S2. Mass balance, cellulose and DMU conversion, and PAS product selectivity for the reactions of cellulose with DMU. ${ }^{a}$

\begin{tabular}{|c|c|c|c|c|c|c|c|}
\hline \multirow{2}{*}{$\begin{array}{l}\text { A } \\
\text { entry }\end{array}$} & \multirow[b]{2}{*}{ Solvent } & \multicolumn{6}{|c|}{ mmoles (\% DMU conversion or \% PAS Selectivity) } \\
\hline & & DMU & $\mathrm{DMHI}$ & DMI & $\mathrm{TMHI}$ & TMI & $\begin{array}{l}\text { Total } \\
\text { (PAS) }\end{array}$ \\
\hline 1 & $\mathrm{H}_{2} \mathrm{O}$ & $1.2(51)$ & $0.09(8)$ & $0.18(16)$ & $0.05(4)$ & $0.16(14)$ & $0.48(41)$ \\
\hline 2 & $\mathrm{MeOH}$ & $1.1(49)$ & $0.11(10)$ & $0.23(20)$ & $0.09(8)$ & $0.32(29)$ & $0.75(67)$ \\
\hline 3 & $\mathrm{EtOH}$ & $1.3(58)$ & $0.16(12)$ & $0.27(21)$ & $0.11(9)$ & $0.30(22)$ & $0.84(64)$ \\
\hline 4 & DMI & $1.4(60)$ & $0.07(5)$ & $0.45(33)$ & $0.09(7)$ & $0.54(40)$ & $1.16(85)$ \\
\hline 5 & $\mathrm{DMI} / \mathrm{H}_{2} \mathrm{O}$ & $1.5(63)$ & $0.05(3)$ & $0.18(13)$ & $0.14(10)$ & $0.32(22)$ & $0.68(48)$ \\
\hline B & \multicolumn{7}{|c|}{ mmoles of carbons in PAS from cellulose } \\
\hline entry & Solvent & & DMHI & DMI & $\mathrm{TMHI}$ & TMI & total \\
\hline 1 & \multicolumn{2}{|l|}{$\mathrm{H}_{2} \mathrm{O}$} & 0.18 & 0.36 & 0.14 & 0.48 & 1.16 \\
\hline 2 & \multicolumn{2}{|l|}{$\mathrm{MeOH}$} & 0.23 & 0.45 & 0.27 & 0.9 & 1.91 \\
\hline 3 & \multicolumn{2}{|l|}{$\mathrm{EtOH}$} & 0.32 & 0.54 & 0.34 & 0.89 & $2.09(15)$ \\
\hline 4 & \multicolumn{2}{|l|}{ DMI } & 0.14 & 0.91 & 0.27 & 1.63 & $2.95(31)$ \\
\hline 5 & \multicolumn{2}{|l|}{$\mathrm{DMI} / \mathrm{H}_{2} \mathrm{O}$} & 0.09 & 0.36 & 0.41 & 0.95 & $1.82(11)$ \\
\hline C & \multicolumn{7}{|c|}{ mmoles of carbons (\% Cellulose Conversion or \% PAS Selectvity) } \\
\hline entry & \multicolumn{2}{|c|}{ Solvent } & Cellulose & Sorbitol & Glycerol & PG & EG \\
\hline 1 & \multicolumn{2}{|l|}{$\mathrm{H}_{2} \mathrm{O}$} & $23.7(32)$ & $7.3(31)$ & $3.3(14)$ & $3.6(15)$ & $5.2(22)$ \\
\hline 2 & \multicolumn{2}{|l|}{$\mathrm{MeOH}$} & $15.6(21)$ & $3.9(25)$ & $1.4(9)$ & $3.7(24)$ & 2.3( \\
\hline 3 & \multicolumn{2}{|l|}{$\mathrm{EtOH}$} & $14.1(19)$ & $5.5(23)$ & $0.7(5)$ & 2.7 (19) & $1.8(13)$ \\
\hline 4 & \multicolumn{2}{|l|}{ DMI } & $9.6(13)$ & $2.5(26)$ & $0.96(10)$ & $1.1(11)$ & $1.3(13)$ \\
\hline 5 & \multicolumn{2}{|c|}{$\mathrm{DMI} / \mathrm{H}_{2} \mathrm{O}$} & $16.3(22)$ & $5.2(32)$ & $2.1(13)$ & $2.0(12)$ & $2.4(15)$ \\
\hline \multicolumn{8}{|c|}{$\begin{array}{l}\text { asigmacell cellulose }(2.0 \mathrm{~g}) \text {, DMU }(0.2 \mathrm{~g}, 2.23 \mathrm{mmol}), \mathrm{Ru} / \mathrm{C}(0.2 \mathrm{~g}) \text {, and solvent }(20 \mathrm{ml}) \text { were } \\
\text { heated in a } 75 \mathrm{ml} \text { stainless steel autoclave at } 200{ }^{\circ} \mathrm{C} \text { in two steps: first, under } \mathrm{N}_{2} \text { inert } \\
\text { atmosphere ( } 35 \mathrm{bar}) \text { for } 6 \text { hours, then } \mathrm{H}_{2}(30 \text { bar) for } 6 \text { hours. A) DMU conversion and PAS } \\
\text { selectivities are calculated. Selectivity is measured by HPLC/UV with benzyl alcohol solution } \\
(5.0 \mathrm{mM}) \text { as an internal standard. B) mmoles of carbons recovered in PAS from cellulose. C) } \\
\text { Cellulose conversion and carbon recovered (mmoles) in polyols. Polyol selectivity were } \\
\text { measured by HPLC/RID calibrated with cis- } 1,5 \text {-cyclooctane diol ( } 10 \mathrm{mM}) \text { as an internal } \\
\text { standard. b, cDMU and cellulose converted. Selectivity is reported in parentheses. }\end{array}$} \\
\hline
\end{tabular}


Table S3. Mass balance, sugar and DMU conversion, and PAS product selectivity for the reactions of sugars with DMU. a

\begin{tabular}{|c|c|c|c|c|c|c|c|}
\hline \multirow{2}{*}{$\begin{array}{l}\text { A } \\
\text { ent }\end{array}$} & \multicolumn{7}{|c|}{ mmoles (\% DMU conversion or $\%$ PAS selectivity) } \\
\hline & Sugar & Solvent & DMUb & DMHI & DMI & TMHI & TMI \\
\hline 1 & Fructos & Water & $1.8(74)$ & $0.045(3$ & $0.16(10)$ & $0.14(8)$ & $0.43(26)$ \\
\hline 2 & Glucose & water & 1.7( & $0.068(4$ & $0.48(28)$ & $0.09(5)$ & $0.18(11)$ \\
\hline 3 & Fructos & $\mathrm{MeOH}$ & $1.6(72)$ & $0.14(8)$ & $0.27(17)$ & $0.11(7)$ & $0.52(32)$ \\
\hline 4 & Glucose & $\mathrm{MeOH}$ & $1.7(76)$ & $0.09(5)$ & $0.64(37)$ & $0.05(3)$ & $0.34(20)$ \\
\hline 5 & Fructos & $\mathrm{EtOH}$ & $1.75(77)$ & $0.07(4)$ & $0.43(25)$ & $0.20(12)$ & $0.57(33)$ \\
\hline 6 & Glucose & $\mathrm{EtOH}$ & $1.7(75)$ & $0.20(12$ & $0.70(41)$ & $0.16(9)$ & $0.25(15)$ \\
\hline 7 & Fructos & DMI & $1.97(87)$ & $0.11(6)$ & $0.36(18)$ & $0.05(2)$ & $1.2(61)$ \\
\hline 8 & Glucose & DMI & $1.84(81)$ & $0.27(15$ & $0.66(36)$ & $0.09(5)$ & $0.48(26)$ \\
\hline 9 & Fructos & $\mathrm{DMI} / \mathrm{H}_{2} \mathrm{O}$ & $1.8(79)$ & 0.09 (5) & $0.18(10)$ & $0.20(11)$ & $0.41(23)$ \\
\hline 10 & Glucose & $\mathrm{DMl} / \mathrm{H}_{2} \mathrm{O}$ & $1.93(85)$ & $0.20(11$ & $0.48(25)$ & $0.05(2)$ & $0.25(13)$ \\
\hline
\end{tabular}

B mmoles of carbons in PAS from glucan

\begin{tabular}{llllll} 
& & DMHI & DMI & TMHI & TMI \\
\hline 1 & Fructos Water & 0.09 & 0.32 & 0.41 & 1.3 \\
2 & Glucose water & 0.14 & 0.95 & 0.27 & 0.54 \\
3 & Fructos $\mathrm{MeOH}$ & 0.27 & 0.54 & 0.34 & 1.57 \\
4 & Glucose $\mathrm{MeOH}$ & 0.18 & 1.27 & 0.14 & 1.02 \\
5 & Fructos EtOH & 0.14 & 0.86 & 0.61 & 1.70 \\
6 & Glucose EtOH & 0.41 & 1.41 & 0.48 & 0.75 \\
7 & Fructos DMI & 0.23 & 0.73 & 0.14 & 3.61 \\
8 & Glucose DMI & 0.54 & 1.32 & 0.27 & 1.43 \\
9 & Fructos DMI/ $\mathrm{H}_{2} \mathrm{O}$ & 0.18 & 0.36 & 0.61 & 1.23 \\
10 & Glucose DMI/ $\mathrm{H}_{2} \mathrm{O}$ & 0.41 & 0.95 & 0.14 & 0.75 \\
\hline $\mathrm{C}$ & mmoles of carbons (\% sugar conversion or \% polyol selectivity)
\end{tabular}

\begin{tabular}{|c|c|c|c|c|c|c|c|}
\hline entry & & Solvent & Glucan $^{c}$ & Sorbitol & Glycerol & $P G$ & EG \\
\hline 1 & Fructos & Water & $33.3(100)$ & 10.7 & & $7.3(22)$ & $5.3(16)$ \\
\hline 2 & Glucose & water & $33.3(100)$ & & & $4.3(13)$ & $.0(30)$ \\
\hline 3 & Fructos & $\mathrm{MeOH}$ & $33.3(100)$ & $9.3(28)$ & $1.0(3)$ & $7.7(23)$ & $5.3(16)$ \\
\hline 4 & Glucose & $\mathrm{MeOH}$ & $33.3(100)$ & $8.0(24)$ & $2.3(7)$ & 5.7 (17) & $9.7(29)$ \\
\hline 5 & Fructos & EtOH & $33.3(100)$ & $6.3(19)$ & $5.0(14)$ & $8.3(25)$ & $6.0(18)$ \\
\hline 6 & Glucose & $\mathrm{EtOH}$ & $33.3(100)$ & $7.0(21)$ & $3.0(9)$ & $4.0(12)$ & $11.3(34)$ \\
\hline 7 & Fructos & DMI & $33.3(100)$ & $8.3(25)$ & $1.7(5)$ & $10.7(32)$ & 3.7 (11) \\
\hline 8 & Glucose & DMI & $33.3(100)$ & $8.0(24)$ & $2.7(8)$ & $5.3(16)$ & $9.0(27)$ \\
\hline 9 & Fructos & $\mathrm{DMl} / \mathrm{H}_{2} \mathrm{C}$ & $33.3(100)$ & $9.0(27)$ & $1.7(5)$ & $7.7(23)$ & $5.7(17)$ \\
\hline 10 & Glucose & $\mathrm{DMl} / \mathrm{H}_{2} \mathrm{O}$ & $33.3(100)$ & $8.3(25)$ & $3.0(9)$ & $6.0(18)$ & $8.7(26)$ \\
\hline
\end{tabular}

aSugars $(1.0 \mathrm{~g})$, DMU $(0.2 \mathrm{~g}, 2.23 \mathrm{mmol}), 0.1 \mathrm{~g} \mathrm{Ru} / \mathrm{C}(5 \mathrm{wt} \%)$, and $20 \mathrm{ml}$ solvent were heated in a $75 \mathrm{ml}$ stainless steel autoclave at $200{ }^{\circ} \mathrm{C}$ in two steps: first, under $\mathrm{N}_{2}$ inert atmosphere (35 bar) for 6 hours, then $\mathrm{H}_{2}$ (30 bar) for 6 hours. A) DMU conversion and PAS selectivities are calculated. Selectivities are reported in parentheses and analyzed by HPLC/UV with benzyl alcohol solution $(5.0 \mathrm{mM})$ as an internal standard. B) mmoles of carbons recovered in PAS from sugars. C) Sugar conversions and mmoles of carbons recovered in polyols. Polyol 
selectivities were analyzed by HPLC/RID calibrated with cis-1,5-cyclooctane diol (10 mM) as an internal standard. ${ }^{b, c}$ Converted DMU and glucan.

Table S4. Results of coupling reactions of 1,2-PG and DMU at various temperature. ${ }^{a}$

\section{Time Temp TMHI \%}

\begin{tabular}{cccc}
\hline 1 & 24 & 80 & 0 \\
\hline 2 & 12 & 120 & 10 \\
\hline 3 & 12 & 150 & 32 \\
\hline 4 & 12 & 180 & 79 \\
\hline 5 & 12 & 200 & 81
\end{tabular}

a1,2-PG (0.15 g, $14 \mathrm{mmol}), \mathrm{DMU}(0.088 \mathrm{~g}, 7 \mathrm{mmol}), 0.2 \mathrm{~g} \mathrm{Ru} / \mathrm{C}(5 \mathrm{wt} \%)$, and $20 \mathrm{ml} 2-\mathrm{MeTHF}$ were heated in a $75 \mathrm{ml}$ stainless steel autoclave under inert atmosphere $\left(\mathrm{N}_{2}, 35\right.$ bar $)$ at 200 ${ }^{\circ} \mathrm{C}$. Yields were analyzed by ${ }^{1} \mathrm{H}-\mathrm{NMR}$ with naphthalene as an internal standard.

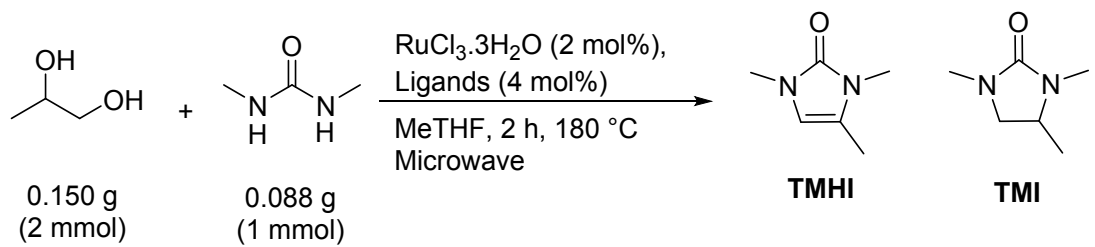

Table S5. Results of in situ formation of homohgeneous Ru catalysts with varying phosphine ligands. ${ }^{a}$

\begin{tabular}{ccccc}
\hline Entry & Ligand & bTMHI \% & cDiol \% & cDMU \% \\
\hline 1 & 38 & 19 & 44 \\
\hline 2 & 44 & 30 & 60 \\
\hline 3 & & 51 & 56 & 82 \\
\hline 5 & & 23 & 23 & 56
\end{tabular}

${ }^{a R u C l} \cdot 3 \mathrm{H}_{2} \mathrm{O}(0.02 \mathrm{mmol})$, phosphine ligands (0.04 mmol), 1,2-PG $(0.15 \mathrm{~g}, 2 \mathrm{mmol})$, DMU $(0.088 \mathrm{~g}, 1 \mathrm{mmol})$, and 2-MeTHF $(1.5 \mathrm{ml})$ were heated in a $10 \mathrm{ml}$ microwave vial under $\mathrm{N}_{2}$ inert atmosphere at $200^{\circ} \mathrm{C}$ for 2 hours. ${ }^{b} Y i e l d s$ and 'selectivity was analyzed by ${ }^{1} \mathrm{H}-\mathrm{NMR}$ with naphthalene as an internal standard. 
III. Figure S1. ${ }^{1} \mathrm{H}$ NMR of the oxidation reaction of 1,2-propylene glycol into hydroxyacetone
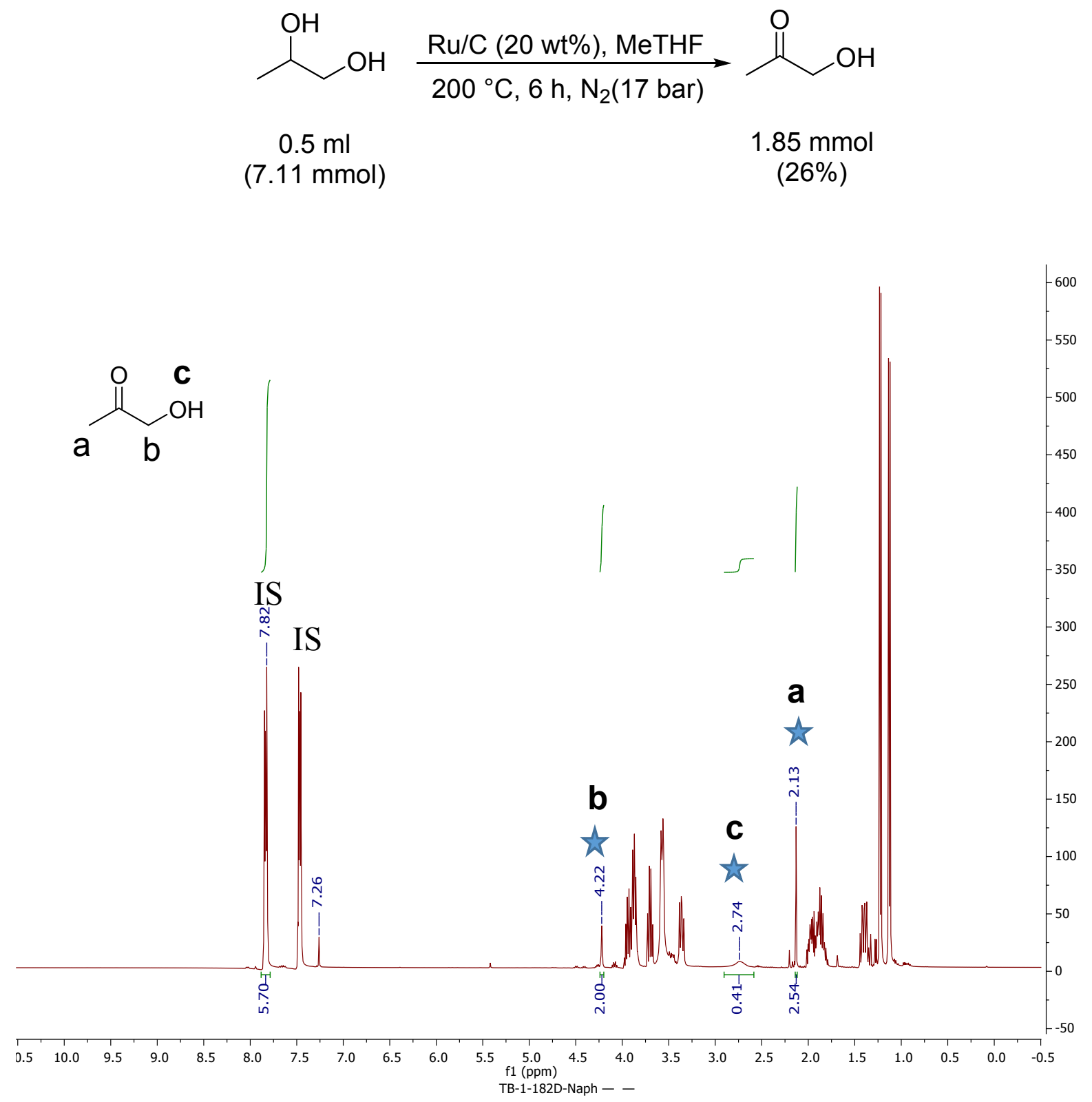